Article

\title{
An IFC Interoperability Framework for Self-Inspection Process in Buildings
}

\author{
José L. Hernández ${ }^{1}$ (D), Pedro Martín Lerones ${ }^{1, *}$, Peter Bonsma ${ }^{2}$, Andrè van Delft ${ }^{3}$, \\ Richard Deighton ${ }^{3, *}$ and Jan-Derrick Braun ${ }^{4}$ \\ 1 Fundación CARTIF, Parque Tecnológico de Boecillo, 205, 47151 Boecillo, Valladolid, Spain; josher@cartif.es \\ 2 RDF Ltd., 1320 Bankya, P.O. Box 32, Bulgaria; peter.bonsma@rdf.bg \\ 3 Demo Consultants, Postbus 642, 2600 AP Delft, The Netherlands; andre@demobv.nl \\ 4 Hotchief, Opernplatz 2, 45128 Essen, Germany; Jan-Derrick.Braun@hochtief.de \\ * Correspondence: pedler@cartif.es (P.M.L.); richard@demobv.nl (R.D.)
}

Received: 22 September 2017; Accepted: 12 February 2018; Published: 17 February 2018

\begin{abstract}
Self-inspection of buildings is the process of controlling the quality of construction work in order to ensure that the specifications are implemented according to the design. Under current practices, self-inspection is totally dependent on the operator's skills, being a process that can be time-consuming and sometimes difficult to achieve. The Intuitive Self-Inspection Techniques using Augmented Reality (INSITER; for construction, refurbishment and maintenance of energy-efficient buildings made of prefabricated components) project aims to develop a software tool to support construction workers in self-inspection processes, with the overall objective of reducing major errors and extra costs. Nevertheless, one of the challenges is the lack of interoperability between the various equipment used to carry out self-inspection. Devices and current tools deployed on-site do not speak the same language, which leads to a lack of communication. Therefore, this paper presents a framework under which the equipment would be able to send information in a common format. For this purpose, the Industry Foundation Classes (IFC) de-facto standard has been established as a viable data model to represent all the information related to the building project. Along these lines, Building Information Modeling (BIM) information and IFC-compliant databases have been designed for the representation of data coming from Computer-Aided Design (CAD) modeling, laser scanning, thermography and sensor networks. Besides the IFC-data repositories, the framework is a multi-layer architecture with the goal of ensuring interoperability and promoting the stakeholders' objectives for self-inspection during the entire construction process.
\end{abstract}

Keywords: self-inspection; building quality; prefabricated components; energy-efficient buildings; Building Information Modelling (BIM); Industry Foundation Classes (IFC); Augmented Reality (AR)

\section{Introduction}

In Europe, a critical mass of Energy-efficient Buildings (EeB) will be achieved by 2020 through sustainable industrialization of high-performance architectural, structural and building-service components [1]. However, realizing the targeted performance in design is hampered by critical shortcomings during on-site construction and refurbishment, which leads to lower quality and sub-optimal energy saving during the building lifecycle. The Intuitive Self-Inspection Techniques (INSITER; using Augmented Reality (AR), for construction, refurbishment and maintenance of energy-efficient buildings made of prefabricated components) project [2], strives to reduce the discrepancy in quality and energy performance between design and practice. It is well known that a large portion of 'failure costs' can be contributed to construction errors [1]. There are several causes of construction errors, such as calculation errors, leakages and incorrect assembly, among others. 
On occasion, these errors could have been easily avoided, while in other cases errors are far more difficult to prevent. For instance, Building Information Modelling (BIM)-based construction projects allow for easier management of materials and avoid changes to materials. Usually, these errors imply high repair costs, or when left unattended to, they can have large, negative consequences on the building's technical, and subsequently, financial performance.

The INSITER project [2] aims to eliminate the gaps in quality and energy performance between design and realization of $\mathrm{EeB}$, based on prefabricated components, by applying BIM with intuitive and cost-effective AR, which connects the virtual building model and the physical building (or the building under construction) in real-time. In order to prevent errors and work accurately, INSITER has developed a new methodology for self-instruction and self-inspection by construction workers, subcontractors, component suppliers, and other stakeholders during on-site working processes, which is supported by a coherent set of hardware and software tools.

However, the concept of BIM is applied and understood in many ways (e.g., a simple information repository, a complete data model, etc.) [3]. In the design, construction and maintenance phases, there is no single BIM model that is used the same way, by all the stakeholders. This also applies for self-instruction, and therefore BIM application needs to be adjusted for the self-instruction process. In practice, common BIM models are too complicated for this purpose; there is a plethora of information which is not structured in a step-by-step process for on-site construction workers. It is difficult to inspect details and technical quality by comparing the BIM model and the realized building.

The data for comparison with the BIM models is acquired via three-dimensional (3D) measurement devices adjusted for self-inspection. In practice, there are issues with robustness, and user-friendliness, as well as time and cost effectiveness for applying high-tech 3D laser scanning, thermal imaging and acoustic measurement, during on-site working processes [4,5]. Aside from the aforementioned difficulties, other challenges also arise in the form of differences in geolocations, building typologies and materials, working cultures, methodologies, software tools and data registration.

The main challenge in developing a toolset to support the self-inspection process is the implementation of the appropriate interoperability standards for data exchange in buildings. Industry Foundation Classes (IFC), an open industry standard format, has come into play for this purpose. Under the INSITER approach, based on the IFC standard [6], the various necessary data and information can be merged to provide useful data; the combination of all these resources improves the construction process by reducing the costs and time, while concurrently and preemptively detecting potential errors.

\section{Self-Inspection Concept}

Quality management is a crucial component of all projects, especially regarding the overall project performance. Put simply, performed tasks have to be verified in a timely manner to avoid follow-up problems that might multiply, and inevitably lead to critical errors. A well-defined self-inspection process is key to avoiding these critical errors.

What is self-inspection? In short, it is a checklist with fixed instructions, which enables workers to evaluate, or double-check all specified working steps for inspection. If any issues are detected, it is possible to solve them at a very early stage of the process. Since the checklist considers the most common errors, avoiding those errors will lead to reliable working results and prevent small errors from leading to critical issues.

With clear self-inspection protocols, there is no need to involve additional personnel. Site-workers will be enabled to check the progress by themselves. By checking several small issues according to a checklist, the worker will be able to control the generic working-step, saving time and reducing the cost of the project.

Additionally, systematic progress verification checkpoints will allow for a clear overview of project progress, and therefore automatically enhance transparency and quality for all stakeholders. 
Regular progress verification enables the project management to perform valuable progress monitoring and efficiently fulfill quality-management requirements.

With respect to the current practices, there is no methodology under which the self-inspection procedures are applied in the construction of buildings. Generally speaking, the existing framework is based on sets of checklists according to the national or regional regulations to ensure certain quality parameters. Although technologies and tools are used to perform building commissioning, their data are not usually connected and, thereby are less efficient. Finally, these techniques are normally employed at the end of the construction process, however, the proposed framework would allow the use of BIM to cover the whole building life-cycle.

\section{Interoperability Standards for Data Exchange in Buildings}

Within the world of Information Technologies (IT), there is an ever-growing number of information exchange standards, each with its own purpose and scope. The INSITER project selected standards take into account the possibility that existing standard(s) may not fulfil the needs and requirements, and could possibly require the combination of several standards.

Mature and often-used exchange formats, such as DraWinG (DWG), AutoCAD and Revit native file formats, are closed (or proprietary) formats which introduce a major dependency on one software vendor. Standard alternatives are also available, like Drawing Exchange Format (DXF) and Green Building Extensible Markup Language (gbXML) [7], however, both standards have a much more limited scope. For instance, with gbXML, the scope is only related to energy performance. For many requirements of the INSITER framework, this is not sufficient. Consequently, the standard IFC [6] was the most relevant candidate for INSITER selection. However, the question remains, does it cover all requirements? Although IFC is very complete, the INSITER framework is still required to offer support for multiple file formats, as well as dedicated semantic structures.

Concerning data exchange standards, there are three main characteristics:

- $\quad$ Is it an open or closed standard? There are different stipulations, but in this context, we call a standard open if the specification is well defined and available for anybody to implement.

- The scope of the standard. What type of content or knowledge can be carried, and what purpose does exchanging such content fulfil?

- The maturity of the standard, here we find at least two levels. The first level is in regard to the technical maturity. Does the structure provide enough freedom to carry what needs to be exchanged, and is it restrictive enough to prevent ambiguity on the receiving side? The second level concerns practical use. Is the standard available in the relevant software packages, and is the quality of support for the standard enough for practical use?

For projects like INSITER, and within the building and construction industry, the choice regarding exchange standards is straightforward. There is only one open standard (IFC, also called ISO16739) that is widely accepted, mature and covers most of the disciplines within the sector $[6,8]$.

As mentioned above, IFC was chosen for the INSITER framework. Throughout the years several versions of IFC have been created. The initial version with real practical success was published as version IFC2x3 TC1 (first technical corrigendum) by the BuildingSMART project (H2020). It has not only been implemented in research pilots, but has also been frequently used by professionals. This success has seemingly delayed the major improvements that have come with more recent releases. IFC4 [8] has been available for several years (with two official updates, i.e., IFC4 ADD1 and IFC4 ADD2); still, the majority of exchange through IFC is done with version IFC2x3. Also, the follow-up of IFC4 (i.e., IFC4x1) is very close to official release, or has likely already been released at the time of paper publication.

IFC4 [8] has many new features of importance for selection by INSITER, including key defining factors for being selected. These factors include, but are not limited to: 
- Improved representation of curves and surfaces through non-uniform rational B-spline (NURBS), B-Spline and Bezier algorithm definitions (Rational).

- Improved scheduling capabilities, such as storing content representing scheduling information very close to the capabilities or freedom available with commercial packages like Microsoft Project.

- $\quad$ Usable texture embedding capabilities.

- $\quad$ Newer, more compressed ways of storing geometrical data through vertex index arrays and sets of triangles, with or without normal information.

Several of the above features are already available in IFC2x3 TC1, although they are not as maturely defined, which has proven to be cumbersome, and sometimes even impossible for the INSITER framework.

IFC4x1 adds support for geometrical representations often used within Geographical Information Systems (GIS) like Clothoid curves. These representations are grouped together and sometimes also name the alignment extensions (most likely, IFC4x1 will contain Version 1.1 of these alignment extensions). This will enable support for bridge, road, rail and tunnel representations, however, most of these extensions are still ongoing and may become part of IFC4x2.

Within INSITER, the extensions added to the later versions of IFC fit very well for the purpose of the framework. Unfortunately, embedding the newer versions of the open IFC standard is progressing slowly, and current software often has utilized the older, but successful, IFC2x3 TC1 version, rather than IFC4 (ADD1/2) or IFC4x1. Therefore, INSITER will help the implementers of the open standard in pushing these great new functionalities. Currently, a population of existing IFC4 files, with one or more different embedded textures, can be used as working examples to assist software companies with populated data which has not yet been explored.

\section{Proposed Framework}

As stated, self-inspection tools support the evaluation of building construction and energy performance, and require data to assess these facilities. Additionally, multiple data formats are available for this information, which needs to be harmonized into IFC, as previously mentioned. Nevertheless, these two contexts converge within the BIM concept. On the one hand, BIM makes use of IFC4 as one of the standards for data exchange, and on the other hand, it covers the entire construction process, allowing for both self-inspection and even self-instruction. However, these two worlds need to be connected and additional data is necessary. The proposed framework is shown in Figure $1[9,10]$.

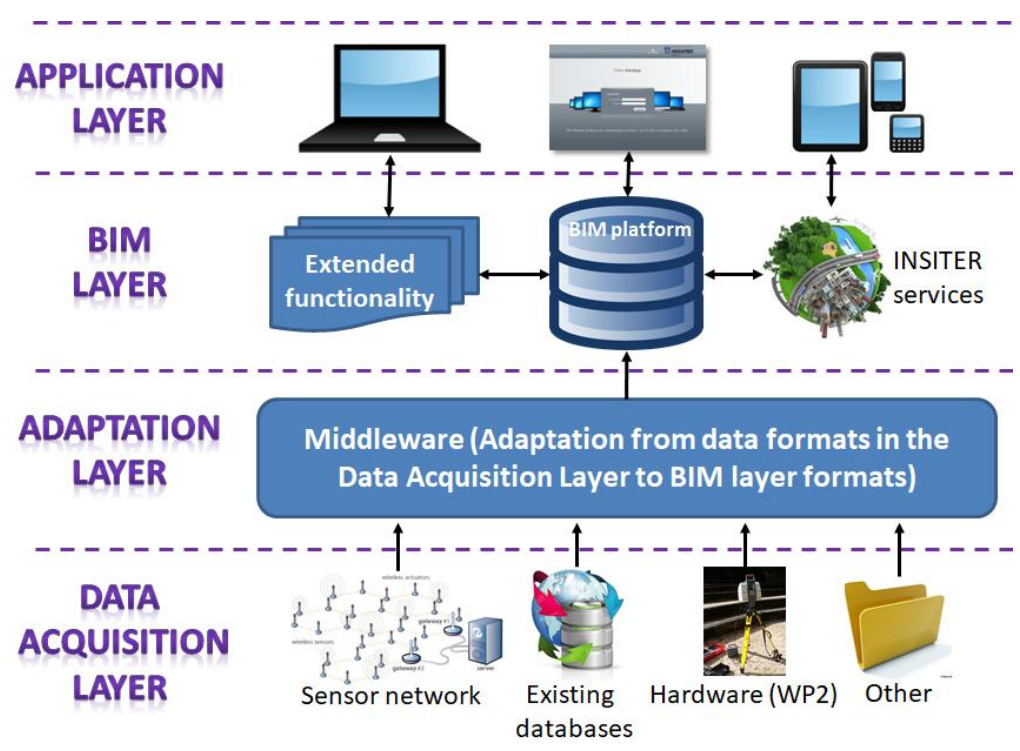

Figure 1. Architecture framework for the self-inspection tools based on Industry Foundation Classes (IFC). 
Explaining the framework from the bottom layer up, the data needs to be acquired (Data Acquisition Layer). In this case, information from hardware for self-inspection is obtained. The hardware and how its information is integrated are explained below. Laser scanner, thermography, and sound are the main sources for quality checks. Moreover, sensor networks are included, which provide real and periodic data about the performance of the systems during the maintenance stage. Other data may be obtained from existing databases of buildings, documents, data-sheets and BIM models, among others.

The next layer (Adaption Layer), is dedicated to harmonize all of the information in order to exchange the data in the same format. Since IFC is used for the INSITER format, the IFC information representation is related to the BIM models, hardware acquisition systems and dynamic data (i.e., sensors). Data-sheets and documents are not translated, although they do extend the IFC information.

The main layer in the INSITER architecture framework is the BIM layer, where the data repositories are represented. In the case of BIM models, the open source BIM Server is selected. Primarily, this is to provide the source code and possible adaptations of the repository, to avoid any modification of other repositories. The second repository is the database, whose scheme is developed according to the IFC standard and is IFC-compliant. Then, it may be viewed as an extension of the BIM Server to contain dynamic data. While BIM repository contains the static information of the building, databases are designed for the collection of dynamic data from sensors. Thus, the combination of both databases enables the hardware systems to provide useful information about the performance of the building during any step of the construction process. Finally, the sharepoint is the third element to make up the INSITER server environment. It stores documents, data-sheets and other relevant information, in order to produce the methods and procedures for self-inspection.

After all of this information is merged and stored in a common language (i.e., IFC), the next step is the development of services. While some services are implemented within the BIM layer (e.g., model checks) because they may be integrated into the BIM Server with simple scripts, the most important services are deployed under the top-level of the architecture (Application Layer). The Application Layer integrates information from the applications that check building quality, such as planning and cost tools, local monitoring visualizers, or BIM viewers. It allows the construction workers to perform quality analysis of the building by extracting information from the BIM model, as well as performance monitoring on dashboards and interaction with experts via remote communication, effectively cross-checking solutions in a timely manner.

\section{Data Integration Mechanisms}

One of the challenges facing building self-inspection is interconnecting the different measurement instruments with BIM [11]. This is an interoperability challenge due to the multiple formats that need to be combined in the common IFC standard. A specific methodology was developed to merge useful digital information for self-inspection purposes and further handling within AutoDesk REVIT (2017 (17.0.503.0), AutoDesk Inc., San Rafael, CA, USA) as BIM worldwide representative software. Suitable digital information sources are 3D point clouds (acquired by laser scanning or photo-based scanning), and 2D images (provided by thermal cameras and sound brushes) [12]. The integration was field-tested at the CARTIF-3 building (INSITER Spanish demonstrator), for inspection tasks directly linked to long-term maintenance planning.

3D laser scanning of representative elements of this EeB (CARTIF-3), made of prefabricated modules, were carried out both by a Leica HDS-3000 (time-of-flight (TOF) technology, Heerbrugg, St. Gallen, Switzerland), and a FARO Focus3D X330 (FARO, Lake Mary, FL, USA), in an attempt to evade voids. The data provided by laser scanners consisted of: geometry (XYZ coordinates); color ((red-green-blue; RGB) coordinates (real appearance)); and reflectance (L-index: materials and humidity). Once all of the data was registered, the index of reflectivity was processed according to a tailored algorithm for moisture checking. Finally, XYZ, RGB and L-data were registered together into PolyWorks (2017, Québec, QC, Canada) (a leading universal 3D metrology software solution). 
Thermography (to assess heat gain or loss, and thermal bridges), and acoustic imaging (to assess comfort conditions, such as wasted energy through noise detection), were obtained in parallel. Both types of imaging data were then ready to be mapped on 3D point-clouds in a raster-based process, using MeshLab (an open source system for processing and editing raw data produced by 3D digitization tools or devices, http:/ /www.meshlab.net/) [12]. The resulting enriched 2D/3D blending was saved as a Polygon File Format (PLY) extension, which is a well-known format to describe an object as a collection of vertices, faces, and other elements, along with properties, such as, color and normal direction. As many PLY files that are needed, are created (Figure 2).

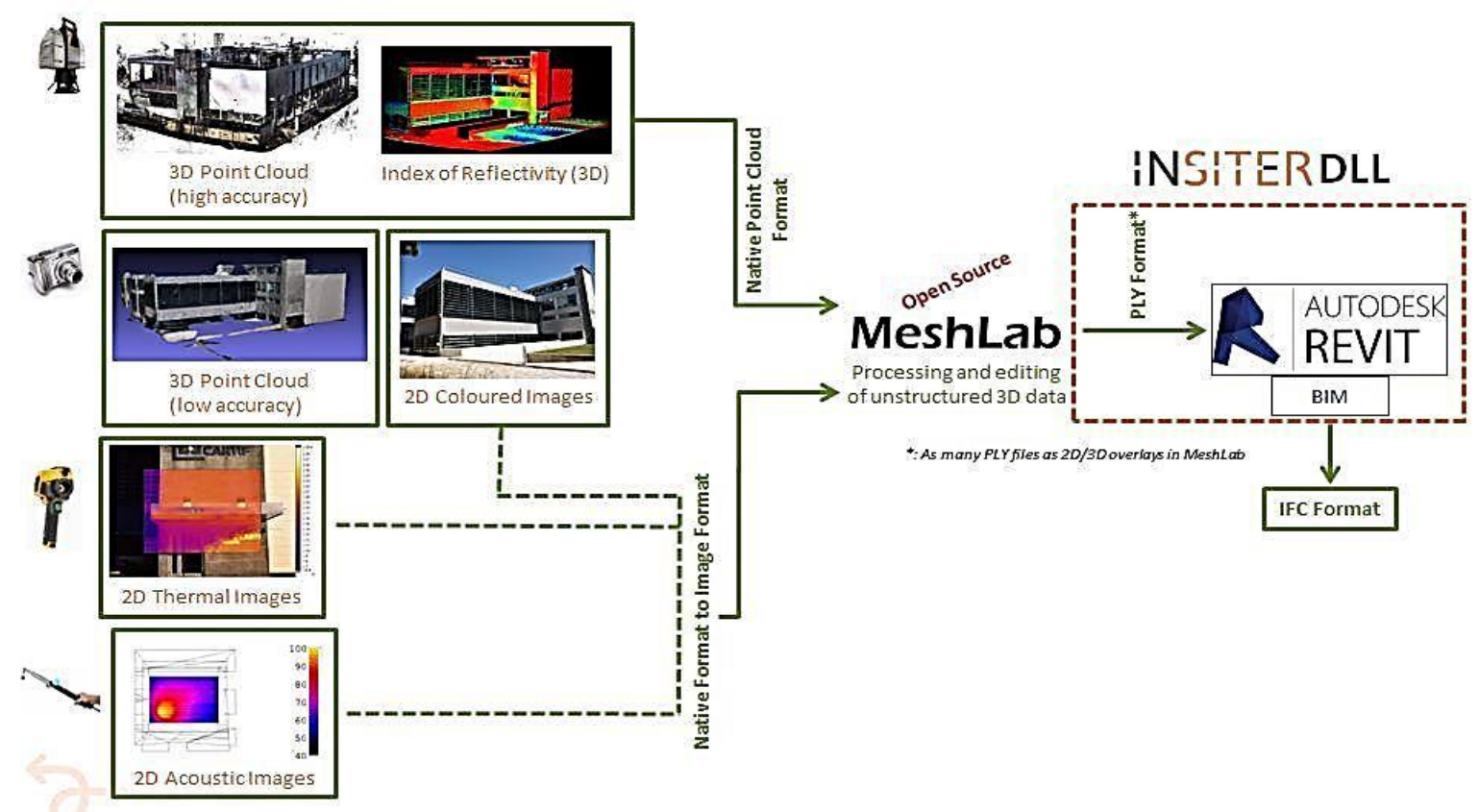

Figure 2. Process for combining 2D and 3D information, for useful for self-inspection of buildings into REVIT.

A specifically tailored plug-in for REVIT has been programmed in $\mathrm{C}++$ and supported by the Point Cloud Library (PCL) as a standalone, large-scale, open software project, for image and point cloud processing. The plug-in is a dynamic-link library (DLL), named INSITER-DLL, and it is able to precisely display the PLY files by layers, in a unique working project (RVT [Revit]/RFA [Revit Families]). A specific toolbar was created to allow management of files that follow this nomenclature:

Base File $(X Y Z):<$ name $>$.PLY

Reflectance (L): $<$ name $>$. $<$ REFLECTANCE $>$.PLY

Colour (RGB): $<$ name $>$. $<$ RGB $>$.PLY

Thermal map: $<$ name $>$. $<$ THERMAL $>$.PLY

Acoustic map: <name $>$. $<$ ACOUSTIC $>$.PLY

These files cover the full range of data needed to assess the operational requirements at the lowest possible energy and financial cost over the entire life-cycle of the building. The user would load the base file into REVIT, and the INSITER-DLL automatically will load all of the related files that meet the requirements previously described. The PLY files serve as 3D templates to extract useful parametric features for self-inspection through fully manual or semi-automatic delineation, or via complementary architecture, engineering and construction (AEC) plug-ins. This process is completed upon export of the parametric objects to IFC from REVIT, as illustrated in Figure 2. 


\section{Energy Efficient Tools for Self-Inspection}

An important goal of the INSITER project is to minimize the energy-performance gap between the "as built" and "as designed" model of the building. The worker on the construction site, who is involved with the verification of the assembly parts and the assembly process itself, must therefore be supported on-site to the maximum extent in order to reach the required level of quality. For this reason, a fully integrated mobile application has been developed [13]. It comprises:

1. A 3D viewer: to view the model "as designed", and compare with "as built".

2. A QR (Quick Response) code reader, for scanning the material codes. Upon detection of a QR code, the associated part of the 3D model will be highlighted, and all relevant information for this component will be accessible to the user.

3. A QR code generator: for digital information exchange with the project leader about any detected pathology.

4. Availability of all required (i.e., instructions, product, project) documents, drawings, movies and images.

5. Fully configurable checklists: to assess the compliance with the project and product requirements.

6. The ability to register observations containing text, pictures, Global Position System (GPS) coordinates, date, time and geographical orientation.

7. A dashboard for monitoring the project Key Performance Indicators (KPIs).

8. AR and Virtual Reality (VR) features.

With the AR features, thermal leakages, humidity and problems with sound insulation can be superimposed on the real building, as seen by the camera on the mobile device. The VR features provide the ability to walk through a 3D model of the building even if the building does not yet exist.

The project leader is provided with a dashboard (as mocked-up in Figure 3) which presents all relevant data of the project, to enable early-stage decision-making.

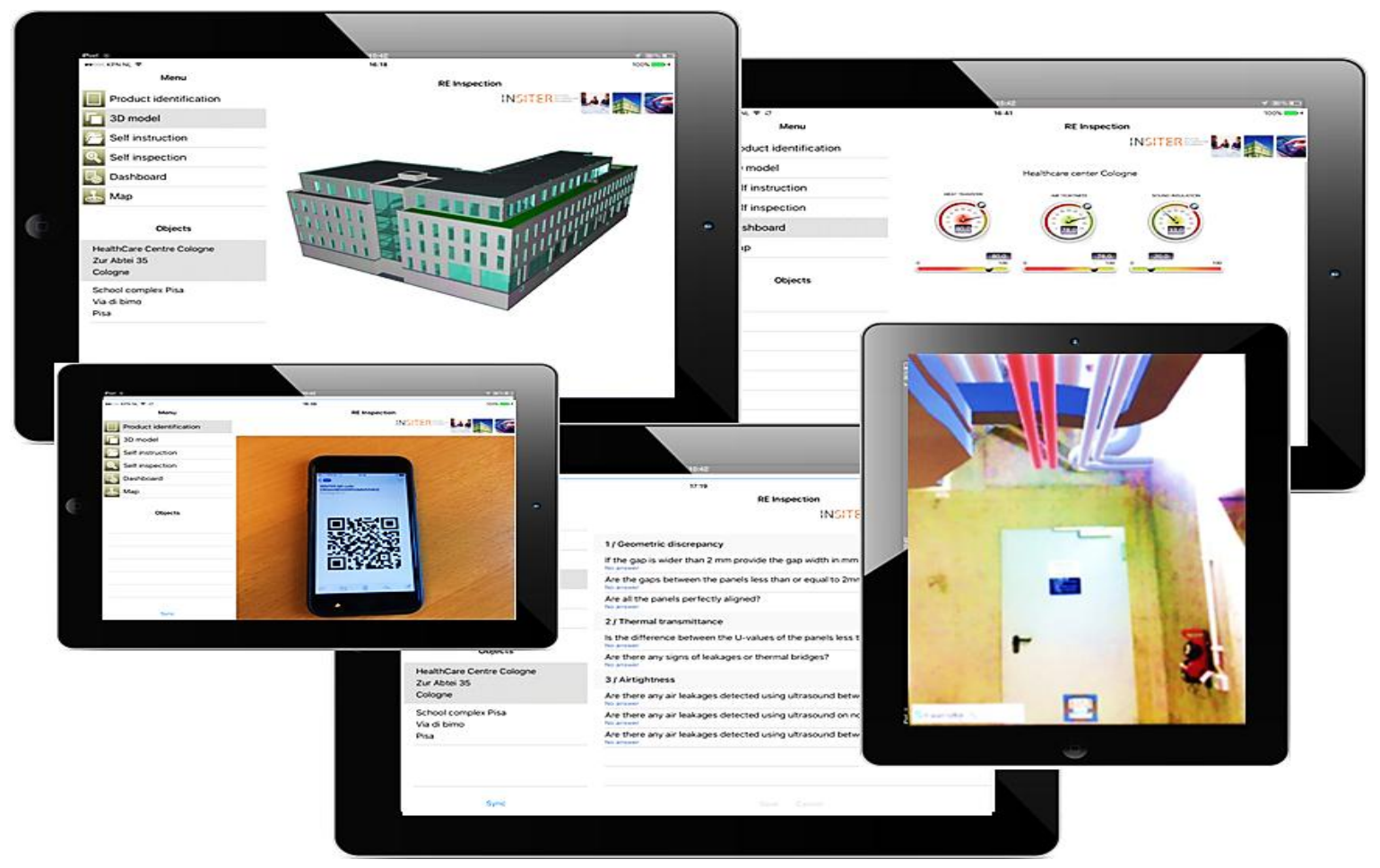

Figure 3. Self-Inspection supported by mobile inspection App. 
The concept of INSITER is to develop a tool that supports the construction work in terms of self-inspecting or self-instructing. For this purpose, several tools are under implementation, and although beta versions are ready, tool developments are still required to solve remaining errors. There is a necessity for further integration in order to run all of the tools together for a holistic implementation. Nevertheless, future trends are in fragmented applications; this is especially true within the building and construction sector. In the automotive, aerospace, and among many other sectors, a 'few' large players are dominating the market and giving strong lead and vision to overall developments.

According to this future trend, the intention of INSITER is to deploy a cloud-based tool linked though a common interface; the access portal will include a set of tools that communicate with the server side in a cloud-based deployment. Then, distributed servers can host the BIM models, including but not limited to, AR reality tools and 3D viewers, with the aim of providing a single access-point to the end-users.

\section{Discussion}

Under the umbrella of the INSITER proposed framework, the intention is to reduce the gaps between the designed and constructed building processes. Mainly, the framework allows for the exchange of BIM information between all of the involved stakeholders, which provides a technical advantage, as all of the workers would be included in the communication loop, with the most-current building data.

This work report on the ability of self-inspection tools to be applied in a harmonized way for the prevention of errors during and after the construction process. The actual practices are mainly based on visual inspection, but leakages or voids in the prefabricated panel joints could still occur. By means of the integration of data acquisition equipment, BIM and self-inspection tools, the errors can be detected during the construction, instead of after the building or structure is completed. The benefit is to reduce the efforts and costs that would be required to solve these common errors. However, interoperability between these tools is still a key challenge which is addressed in the proposed framework. It is important to highlight that the framework does not cover the solution of the errors, but rather is a means to conceptually prevent them.

Moreover, the proposed framework gives technological support to the construction workers, by providing a mechanism for efficient communication of data from on-site and off-site workers. Additionally, the framework allows for connecting virtual and physical buildings in their environments in real-time, with the help of AR tools aimed at ensuring targeted performance.

\section{Conclusions}

Self-inspection is a critical process that improves the quality in the construction process, from design (i.e., BIM models) to maintenance. However, it is usually costly to engage the necessary experts on site. Moreover, the current state-of-the-art technologies require some time to be applied on-site under the existing conditions. All of these factors increase the cost and delay the construction process. These are the main reasons for requiring tools that ease the self-inspection process.

Nevertheless, one pivotal aspect is interoperability. Until now, existing technologies are not able to communicate with each other. Under the INSITER approach, based on IFC standards, the information can be merged in order to provide useful data to high-level applications. Thus, the combination of all these resources improves processes by reducing the cost and time, detecting errors in advance and helping to provide solutions.

The core of this process is the BIM application including complete, and integral building information. The INSITER framework also provides a powerful tool for the management of the entire building process. Deviations, errors in design and/or low performance may be detected in advance due to the holistic digitalization of the information. In this way, BIM validation tools are also integrated in order to assure BIM quality. Hence, clash detection and model validation algorithms work within the proposed framework as BIM services. 
Finally, future work on this framework will finalize the integration of the tools, and will complete applicability-tests in a real-world environment. While the individual implementations and technologies have been tested in controlled environments, the full interoperable INSITER method needs to be applied to a real-world building construction process. This final test-phase has been planned for the final year of the project. Presently, applications of this work have seen a 50\% reduction in energy consumption through the reduction of construction errors, as well as decreased costs (between $20 \%$ and $30 \%$ ) with respect to current practices.

Acknowledgments: The authors would like to thank the rest of partners of the INSITER consortium for their support and help. As well, the authors would like to thank the European Commission for the received funding to carry out this work under the Grant Agreement \#636063.

Author Contributions: José L. Hernández, Andrè van Delft and Peter Bonsma worked on the data formats and framework; Pedro Martín worked on data integration methods; Jan-Derrick Braun worked on the BIM model interoperability; Richard Deighton worked on the development of the energy efficient tool.

Conflicts of Interest: The authors declare no conflict of interest.

\section{References}

1. Directive 2002/91/EC. Available online: http:/ / eur-lex.europa.eu/legal-content/EN/TXT/?uri=CELEX\% 3A32002L0091 (accessed on 14 April 2017).

2. INSITER Project. Available online: https://www.insiter-project.eu/en (accessed on 28 April 2017).

3. Buildingsmart, International openBIM Home. Available online: http://buildingsmart.org (accessed on 9 June 2017).

4. Revel, G.M.; Sabbatini, E.; Arnesano, M. Development and experimental evaluation of a thermography measurement system for real-time monitoring of comfort and heat rate exchange in the built environment. Meas. Sci. Technol. 2012, 23. [CrossRef]

5. Arayici, Y. An approach for real world data modelling with the 3D terrestrial laser scanner for built environment. Autom. Constr. 2007, 16, 816-829. [CrossRef]

6. IFC Overview. Available online: http://www.buildingsmart-tech.org/specifications/ifc-overview (accessed on 7 March 2017).

7. gbXML. Available online: http://www.gbxml.org (accessed on 20 December 2016).

8. IFC4 Release. Available online: http://www.buildingsmart-tech.org/specifications/ifc-releases/ifc4-release (accessed on 8 May 2017).

9. INSITER Consortium. D3.1: Functional Program of Requirements for Planning and Cost; INSITER Consortium: Delft, The Netherlands, 2016.

10. INSITER Consortium. Functional Programme of Requirements for Building Quality and Energy Assessment Software; INSITER Consortium: Delft, The Netherlands, 2016.

11. Chen, K.; Lu, W.; Peng, Y.; Rowlinson, S.; Huang, G.Q. Bridging BIM and building: From a literature review to an integrated conceptual framework. Int. J. Proj. Manag. 2015, 33, 1405-1416. [CrossRef]

12. Lerones, P.M.; Vélez, D.O.; Rojo, F.G.; García-Bermejo, J.G.; Casanova, E.Z. Moisture Detection in Heritage Buildings by 3D Laser Scanning. Stud. Conserv. 2015, 61 (Suppl. 1), 46-54. [CrossRef]

13. Demo Consultants. Re-Suite Software. Available online: http://uk.demobv.nl/downloads/Leaflet_RE_ Suite_UK.pdf (accessed on 5 July 2017). 